\title{
Arguments microbiologiques pour optimiser l'antibiothérapie empirique des cellulites cervico-faciales
}

\author{
Optimization of the empiric therapy of cervicofacial cellulitis: \\ microbiological argued points
}

LUC DUBREUIL, CHRISTEL NEUT

\begin{abstract}
RÉSUMÉ
Le traitement des cellulites vise à éradiquer streptocoques et anaérobies stricts. Lors de traumatismes ou dans les cellulites cutanées, il faudra prendre en compte les staphylocoques. Dans les infections plus sévères, le rôle des entérobactéries est discuté et souvent considéré comme acquis. Dans des situations extrêmes, sur documentation bactériologique, on peut être confronté à la présence de Pseudomonas aeruginosa ou de Staphylococcus aureus méthicilline-résistant.

L'évolution de la résistance des anaérobies stricts à l'amoxicilline (production de ß-lactamase) ou à la clindamycine doit être régulièrement suspectée. Dans les infections sévères des associations, notamment avec les aminoglycosides, sont préconisées. Dans les cellulites circonscrites d'origine dentaire, les associations amoxicilline + métronidazole ou spiramycine + métronidazole sont envisageables. Pour les cellulites cutanées d'extension modérée, on peut utiliser oxacilline + métronidazole, clindamycine \pm métronidazole, amoxicilline + acide clavulanique. Lorsque la cellulite est sévère amoxicilline + acide clavulanique, pipéracilline + tazobactam, céfotaxime ou ceftriaxone + métronidazole sont souvent associés aux aminoglycosides. S'il y a un risque pour le pronostic vital, c'est l'association imipénème + aminoglycoside qui sera retenue. Les cas particuliers et rares, avec des staphylocoques méthicilline-résistants ou de Pseudomonas aeruginosa, sont évoqués. (Med Buccale Chir Buccale 2005; 11: 7-15).
\end{abstract}

mots clés: Anaérobies, infections dentaires, cellulites, association d'antibiotiques

médecine buccale chirurgie buccale

VOL. $11, \mathrm{~N}^{\circ} 1$ 2005

page 7

\section{SUMMARY}

The treatment of cellulitis targets mainly streptococci and anaerobic bacteria. Staphylococci are taken in consideration in case of trauma or cutaneous cellulitis. In more severe infections, the clinician will consider the presence of Enterobacteriaceae that is still debated. The presence of Pseudomonas aeruginosa or meticillin-resistant Staphylococcus aureus may occurs rarely in very severe infections.

Increase resistance to amoxicillin (B-lactamase production) or clindamycin is important among anaerobic bacteria. Antibiotic combinations are needed in severe infections by adding mainly aminoglycosides. In local dental celIulitis the two combinations amoxicillin or spiramycin + metronidazole are useful. In presence of a moderate cutaneous cellulitis oxacillin + metronidazole, clindamycin \pm metronidazole, amoxicillin + clavulanic acid are recommended. The more severe cellulitis are treated by amoxicillin + clavulanic acid, piperacillin + tazobactam, cefotaxime or ceftriaxone + metronidazole combined with aminoglycosides. Facing a life-threatening infection leads to imipenem + aminoglycoside. Rare severe infections involving either a strain of meticillin-resistant Staphylococcus or Pseudomonas aeruginosa is considered herein. (Med Buccale Chir Buccale 2005; 11: 7-15).

key words: Anaerobes, dental infections, cellulites, antibiotic combination 
médecine

buccale

chirurgie

buccale

VOL. $11, \mathrm{~N}^{\circ} 1$ 2005

page 8
L'aide à la décision thérapeutique pour le choix d'un antibiotique repose sur la fréquence des bactéries isolées et leur sensibilité aux différentes familles d'antibiotiques; les autres facteurs primordiaux sont la sévérité de l'infection et l'état du patient.

Dans le cadre des recommandations de l'AFSSAPS [1], il est indiqué que l'étude de la littérature n'a pas permis de mettre en évidence de façon précise les bactéries spécifiques des cellulites. Dans les cellulites aiguës, on retrouve généralement la flore buccale commensale; actinomycètes et staphylocoques sont volontiers associés aux cellulites chroniques.

Les infections de la région cervicale étaient dues jadis à l'oropharyngite mais sa fréquence a décru depuis l'ère des antibiotiques. Maintenant l'origine dentaire est considérée comme la plus fréquente. Les infections des glandes salivaires sont nombreuses, mais on connaît mal la source de l'infection. Les infections traumatiques résultent de l'inoculation de la flore cutanée. On observe une augmentation des infections par injection de drogues IV chez le toxicomane.

Selon Berini et coll. [2], les infections endodontiques sont responsables de $88 \%$ des cellulites de l'adulte. Pour ces auteurs, les $2^{\mathrm{e}}$ et $3^{\mathrm{e}}$ molaires sssont la source des infections de l'espace submyloidien. Pour l'espace sublingual, c'est la première molaire qui est le plus souvent en cause.

\section{Actinomycoses}

Au cours des actinomycoses cervico-faciales aiguës ou chroniques, on rencontre fréquemment Actinomyces israelii, commensal de la plaque dentaire et des caries dentaires. Les autres espèces isolées sont $A$. bovis, $A$. naeslundii; elles sont parfois associées à Actinobacillus actinomycetemcomitans. L'actinomycose survient après traumatisme de la joue ou extraction dentaire. Elle se traduit par une tuméfaction comparable à celle des autre cellulites. La progression de la maladie provoque la décharge d'un pus contenant des grains jaunes, très utiles au bactériologiste pour la mise en évidence des Actinomyces. En effet, la documentation bactériologique de l'actinomycose reste difficile car la culture de ces bactéries est lente. L'idéal est d'isoler la bactérie à partir d'une aspiration à la seringue ou des grains jaunes. La culture conduit à l'obtention de colonies ayant la forme d'une couronne de molaire. La morphologie après coloration de Gram montre la présence de bactéries branchées. Bien souvent, le diagnostic est réalisé en anatomopathologie à partir d'un granulome dentaire péri-apical.

\section{Difficultés de la documentation bacté- riologique}

Dans la plupart de ces infections, les bactéries appartiennent à la flore oropharyngée. Cette flore franchit les barrières physiologiques à la faveur d'une inflammation locale ou d'un traumatisme. Il s'agit d'infections mixtes (aérobies et anaérobies facultatifs + anaérobies stricts), où le taux des anaérobies est très variable selon les études et dépend de la méthodologie utilisée. Afin d'isoler des anaérobies stricts, il convient en effet d'éviter de prélever la flore résidente (préférer la ponction à l'aiguille après désinfection locale), et de réduire au minimum la toxicité de l'oxygène sur le prélèvement durant son transport jusqu'au laboratoire. Un volume supérieur à $2 \mathrm{ml}$, ne nécessite pas de milieu de transport si le prélèvement est acheminé en moins de 6 heures; lorsque le volume est inférieur à $2 \mathrm{ml}$ tout délai supérieur à 30 minutes nécessite l'emploi d'un milieu de transport. En aucun cas le prélèvement ne doit être placé au réfrigérateur. Le choix des milieux de culture et toute la technicité du laboratoire dans le domaine des anaérobies stricts conditionnent la documentation bactériologique, et en particulier la présence d'anaérobies. Les milieux doivent être réduits avant inoculation et incubés en anaérobiose, l'idéal étant la chambre anaérobie. La documentation bactériologique survient dans 18 à $29 \%$ des cas [3]. L'hémoculture s'avère peu rentable: dans une série de 757 patients hospitalisés pour cellulites faciales, $2 \%$ des hémocultures étaient positives avec isolement dans $73 \%$ des cas d'un streptocoque $B$ hémolytique [4].

\section{Bactéries impliquées dans les cellu- lites de la face}

Parmi les bactéries isolées, les streptocoques du groupe milleri (S. constellatus, S. intermedius et 
S. anginosus) sont isolés dans 45 à $66 \%$ des prélèvements [3]. A partir de 110 prélèvements faits en chirurgie maxillo-faciale, Eick et coll. [5] isolent $23 \%$ de bactéries aérobies, $14 \%$ d'anaérobies stricts et $63 \%$ d'infections mixtes. Dans les infections où les aérobies sont présents, ces auteurs isolent toujours un streptocoque, 5 fois un staphylocoque et 8 fois un bacille à Gram négatif. Parmi les bacilles à Gram négatif anaérobies, on observe la prépondérance de Prevotella 148 souches ( 62 souches pigmentées et 86 non pigmentées) et de Fusobacterium (37 souches) parmi les 110 cellulites prélevées.

Parmi les bactéries isolées par Brook [6] dans les abcès de la tête et du cou chez les enfants, on retrouve surtout des streptocoques et des staphylocoques associés à des anaérobies. Les entérobactéries et le Pseudomonas ne sont que très rarement isolés (Tab. 1).

Si on considère de façon plus précise les bactéries isolées en fonction des conditions ayant favorisé le développement de la cellulite, on s'aperçoit que les anaérobies à Gram négatif sont très largement représentés dans les cellulites d'origine dentaires tandis que Staphylococcus aureus est très fréquent lors des traumatismes (Tab. 2). Dans les cellulites d'origine dentaire (Tab. 3), les bactéries prédominantes sont des cocci à Gram + tels que streptocoques, staphylocoques et Peptostreptococcus, associés à des bacilles à Gram négatif anaérobies stricts (Prevotella, Porphyromonas et Fusobacterium) [7]. Une étude en cours de réalisation dans notre laboratoire s'intéresse à la flore des cellulites prélevées par biopsies cutanées. Les dénombrements bactériens sont réalisés avec un seuil de détection de 2 log UFC. g-1 de prélèvement. Les infections cervicales à point de départ buccal sont comparées à des infections abdomino-péritonéales. Les résultats préliminaires rassemblés dans le Tab. 4 montrent que la documentation bactériologique est plus faible pour les cellulites d'origine dentaire: le dénombrement des espèces présentes montre qu'elles sont moins nombreuses et moins variées.

Tableau 1: Bactéries prédominantes isolées dans 67 abcès cervico-faciaux de l'enfant [6]

Predominant aerobic and anaerobic bacteria recovered from 67 children with subcutaneous abscesses of the head and neck

\begin{tabular}{|c|c|c|}
\hline Bactéries & $\begin{array}{l}\text { Abcès de la tête } \\
\qquad(n=31)\end{array}$ & $\begin{array}{l}\text { Abcès du cou } \\
\qquad(N=36)\end{array}$ \\
\hline $\begin{array}{l}\text { Bactéries aérobies ou aéro-anaérobies facultatives } \\
\text { Streptocoques } \alpha \text { hémolytiques } \\
\text { S. pneumoniae } \\
\text { Streptocoque A } \\
\text { Streptocoque B } \\
\text { Streptocoque C } \\
\text { S. aureus } \\
\text { Haemophilus parainfluenzae } \\
\quad \text { Sous total }{ }^{\star}\end{array}$ & $\begin{array}{c}3 \\
1 \\
1 \\
3 \\
\\
8 \\
3 \\
20\end{array}$ & $\begin{array}{c}4 \\
7 \\
1 \\
20 \\
34\end{array}$ \\
\hline $\begin{array}{l}\text { Bactéries anaérobies strictes } \\
\text { Peptostreptococcus spp. } \\
\text { Veillonella spp. } \\
\text { Actinomyces spp. } \\
\text { Propionibacterium acnes } \\
\text { Prevotella spp. } \\
\text { Bacteroides du groupe fragilis } \\
\text { Prevotella pigmentées } \\
\text { Fusobacterium spp. } \\
\quad \text { Sous total }\end{array}$ & $\begin{array}{c}10 \\
3 \\
1 \\
1 \\
10 \\
2 \\
7 \\
5 \\
42\end{array}$ & $\begin{array}{c}7 \\
1 \\
2 \\
\\
3 \\
4 \\
18\end{array}$ \\
\hline Total & 62 & 52 \\
\hline
\end{tabular}

médecine

buccale

chirurgie

buccale

VOL. $11, \mathrm{~N}^{\circ} 1$

2005

page 9 
Tableau 2: Conditions prédisposantes en relation avec les bactéries isolées dans 67 abcès cervico-faciaux [6] Correlation between the predisposing conditions and the bacteria recovered from 67 abscesses.

\begin{tabular}{|c|c|c|c|c|c|c|c|c|c|}
\hline \multirow[t]{2}{*}{$\begin{array}{l}\text { Condition } \\
\text { prédisposante }\end{array}$} & \multirow[b]{2}{*}{$\begin{array}{l}\mathrm{Nb} \text { de } \\
\text { patients }\end{array}$} & \multicolumn{3}{|c|}{$\begin{array}{l}\text { Type de bactéries } \\
\text { ayant cultivées }\end{array}$} & \multicolumn{5}{|c|}{ Bactéries isolées } \\
\hline & & $\begin{array}{l}\text { Aérobies } \\
\text { seuls }\end{array}$ & $\begin{array}{l}\text { Anaérobies } \\
\text { seuls }\end{array}$ & $\begin{array}{l}\text { Aérobies et } \\
\text { Anaérobies }\end{array}$ & S. aureus & S. pyogenes & $\begin{array}{c}\text { Cocci } \\
\text { anaérobies }\end{array}$ & Prevotella & Fusobacterium \\
\hline $\begin{array}{l}\text { Infection dentaire } \\
\text { ou manipulation }\end{array}$ & 9 & & 6 & 3 & 2 & & 7 & 8 & 3 \\
\hline Angine & 6 & & 2 & 4 & 2 & 4 & 4 & 6 & 4 \\
\hline Traumatisme & 13 & 11 & & 2 & 8 & 1 & 1 & 2 & \\
\hline Autres & 8 & & 5 & 3 & 1 & & 4 & 4 & 1 \\
\hline Inconnue & 31 & 24 & 2 & 5 & 15 & 3 & 1 & 3 & \\
\hline Total & 67 & 35 & 15 & 17 & 28 & 8 & 17 & 23 & 9 \\
\hline
\end{tabular}

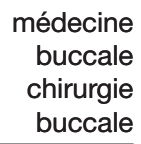

VOL. $11, \mathrm{~N}^{\circ} 1$ 2005 page 10

Tableau 3: Bactéries impliquées dans les cellulites d'origine dentaire [7] Bacteria recovered from cellulitis from dental origin

\begin{tabular}{|l|c|l|c|}
\hline Bactéries aérobies & Nombre & Bactéries anaérobies strictes & Nombre \\
\hline Streptocoques & 9 & $\begin{array}{l}\text { Cocci à Gram + } \\
\text { (Peptostreptococcus) }\end{array}$ & 14 \\
\hline Staphylocoques dorés & 7 & Prevotella spp. & 5 \\
\hline Staphylocoques à coagulase & 1 & Propionibacterium acnes & 3 \\
\hline négative & & Porphyromonas spp. & 2 \\
\hline Entérobactérie & 1 & Fusobacterium sp. & 1 \\
\hline Pseudomonas aeruginosa & 1 & Autres & 7 \\
\hline Total & 19 & & 32 \\
\hline
\end{tabular}

Dans les cellulites abdominales, on rencontre des bactéries fécales (Bacteroides du groupe fragilis, Clostridium, entérocoques) absentes des cellulites d'origine dentaire où l'on retrouve des espèces anaérobies caractéristiques de cette flore $P$. melaninogenica, P. intermedia, Micromonas micros, Eubacterium tenue.

Si l'on considère la plupart des études, on peut mettre en exergue les points suivants:

1 - Les anaérobies stricts sont majoritairement présents avec «quatre mousquetaires » Prevotella, Porphyromonas, Fusobacterium et GPAC (cocci anaérobie à Gram +) ; les Bacteroides du groupe fragilis sont absents.

2 -Association très fréquente avec des streptocoques.
3 -Les bacilles à Gram négatif - E. coli, Klebsiella pneumoniae, Haemophilus influenzae, Pseudomonas aeruginosa - sont rares; on les rencontre plus volontiers chez le patient âgé ou immunodéprimé

4 -Staphylococcus aureus doit être pris en compte chez le toxicomane.

5 -Lors d'infections profondes, on peut isoler des Actinomyces, le bacille tuberculeux et des fungi.

L'antibiothérapie qui sera prescrite devra être active sur les streptocoques et les anaérobies stricts; on pourra en fonction de la sévérité et de l'état du patient, élargir le spectre d'activité aux staphylocoques (traumatisme cutané, toxicomane) et aux enterobactéries. 
Tableau 4: Comparaison des résultats bactériologiques des cellulites selon leur origine (résultats personnels) Compared bacteriological results in relation with the origin of the cellulitis (unpublished results)

\begin{tabular}{|l|c|c|}
\hline Localisation de la cellulite & Cervicale & Abdomino-péritonéale \\
\hline Dénombrement & $4,2 \pm 1,3$ log UFC. $g^{-1}$ & $6,1 \pm 2,1$ log UFC. $\mathbf{g}^{-1}$ \\
\hline Nombre de cultures positives & $6 / 11$ & $18 / 20$ \\
\hline Nombre de bactéries par prélèvement & 2 & 7,1 \\
\hline Bactéries aérobies dont: & 5 & 38 \\
\hline Streptocoques & 3 & 5 \\
\hline Entérocoques & & 10 \\
\hline Staphylocoques & 1 & 1 \\
\hline E. coli & 1 & 14 \\
\hline Entérobactéries & & 5 \\
\hline Pseudomonas aeruginosa & 7 & 1 \\
\hline Bactéries anaérobies strictes dont: & & 89 \\
\hline Bacteroides du groupe fragilis & 2 & 24 \\
\hline Prevotella spp. & & 5 \\
\hline Fusobacterium spp. & & 2 \\
\hline Clostridium spp. & 1 & 12 \\
\hline Eubacterium spp. et Eggerthellaspp. & 2 & 14 \\
\hline Propionibacterium acnes & & \\
\hline GPAC* & & \\
\hline * Cocci anárobie à Gram + & & \\
\hline * aram positive anaeribic cocci & & \\
\hline
\end{tabular}

médecine buccale chirurgie buccale

VOL. $11, N^{\circ} 1$ 2005

page 11

\section{Sensibilité des anaérobies aux anti- biotiques}

La résistance des anaérobies a évolué et la production de $B$-lactamase remet en cause l'utilisation en monothérapie de la pénicilline $G$ et des aminopénicillines. Les B-lactamases sont mises en évidence chez Prevotella et Fusobacterium (Tab. 5) [8-11]. Au moins $30 \%$ des Prevotella d'origine dentaire produisent une B-lactamase. Ces pourcentages pourraient augmenter dans la mesure où des taux plus élevés sont observés dans d'autres types de prélèvements [12]. Si l'on considère les souches produisant une B-lactamase, les CMI de l'amoxicilline sont toujours supérieures à $0,5 \mathrm{mg}$. $\mathrm{l}^{-1}$ et peuvent atteindre des valeurs élevées de l'ordre de $128 \mathrm{mg}^{\mathrm{I}^{-1}}$. Cependant les CMI de l'amoxicilline sont souvent comprises entre 1 et $16 \mathrm{mg}$. $\mathrm{I}^{-1}$. Il est possible qu'une partie de ces souches aient pu être éradiquées par l'amoxicilline si les doses étaient suffisamment élevées et si l'inoculum bactérien était faible. Actuellement, toute souche de Prevotella produisant une B-lactamase est considérée comme résistante aux aminopénicillines, aux céphalosporines de première génération, au céfuroxime, cefpodoxime, céfixime et céfotiam [13]. La production de B-lactamase chez Fusobacterium reste comprise entre 5 et $10 \%$.

La production de $ß$-lactamase au niveau de la flore buccale est en relation avec les traitements antibiotiques antérieurs. Ainsi, la production de B-lactamase est retrouvée chez $2 \%$ des enfants de 2 mois versus $49 \%$ pour ceux de 24 mois en relation avec la prescription d'antibiotiques [14]. De même chez l'adulte, la production de B-lactamase atteint $38,5 \%$ après un traitement par $B$ lactamine versus $10,9 \%$ après un traitement pour une autre famille d'antibiotique. Plus la durée du traitement augmente plus la production de B-lactamase est fréquente [15]. 
Tableau 5: Taux de production des B-lactamases chez les bacilles à Gram négatif anaérobies stricts Rates of $\beta$-lactamase production among gram-negative anaerobic bacilli

\begin{tabular}{|c|c|c|c|}
\hline Auteurs & Origine des souches & Micro-organismes & $\begin{array}{l}\text { Fréquence de la production } \\
\text { de B-lactamase }\end{array}$ \\
\hline Kuriyama et coll. [8] & & $\begin{array}{l}\text { Prevotella pigmentées } \\
\text { Pr. non pigmentées }\end{array}$ & $\begin{array}{l}35,6 \% \\
31,9 \%\end{array}$ \\
\hline Fosse et coll. [9] & Poches parodontales & $\begin{array}{l}\text { Prevotella spp. } \\
\text { Fusobacterium spp. }\end{array}$ & $\begin{array}{c}30-70 \% \\
0-10 \%\end{array}$ \\
\hline Milazzo et coll. [10] & Parodontites & $\begin{array}{l}\text { Prevotella et } B \text {. ureolyticus } \\
\text { F. nucleatum } \\
\text { Pophyromonas gingivalis } \\
\text { Tannerella forsythia }\end{array}$ & $\begin{array}{c}20 \% \\
7 \% \\
3 \% \\
50 \%\end{array}$ \\
\hline Eick et coll. [11] & Parodontites & $\begin{array}{l}\text { Toutes espèces } \\
\text { Abcès endodontiques }\end{array}$ & $\begin{array}{c}6 \% \\
22 \%\end{array}$ \\
\hline Dubreuil et coll. [12] & Souches non orales & $\begin{array}{l}\text { Prevotella pigmentées } \\
\text { Pr. non pigmentées }\end{array}$ & $\begin{array}{l}52 \% \\
63 \%\end{array}$ \\
\hline
\end{tabular}

médecine buccale chirurgie buccale

VOL. $11, \mathrm{~N}^{\circ} 1$ 2005

page 12

\section{Le traitement antibiotique}

Après drainage chirurgical et débridement des tissus nécrosés, l'antibiothérapie sera empirique compte tenu des délais de la culture bactériologique et du temps nécessaire à l'identification des anaérobies. II s'agit presque toujours d'infections mixtes. Lorsqu'un antibiotique est choisi en première intention, on se demandera s'il est également actif sur les anaérobies à Gram négatif (Prevotella, Porphyromonas et Fusobacterium). Lorsque la réponse est négative le métronidazole est alors ajouté.

Le traitement de l'actinomycose est particulier; il nécessite l'emploi de la pénicilline $\mathrm{G}$ à raison de $20 \mathrm{M} \mathrm{UI}^{\mathrm{j}} \mathrm{j}^{-1}$ pendant 6 semaines, puis relais avec un macrolide ou une tétracycline per os pendant au moins 6 mois.

\section{Infections modérées}

Dans les infections modérées, les antibiotiques employés sont la pénicilline $\mathrm{G}$ par voie intraveineuse ou l'amoxicilline $1 \mathrm{~g}$. x3.j. $\mathrm{j}^{-1}$. La fréquence des B-lactamases chez les anaérobies à Gram négatif fait que l'on associe le plus souvent soit le métronidazole, soit un inhibiteur de B-lactamases. Pour les infections modérées (Tab. 6), il est souhaitable de couvrir streptocoques et anaérobies à Gram négatif. L'association spiramycine- métronidazole, très efficace sur les anaérobies d'origine dentaire [16], peut être préconisée dans les cellulites circonscrites d'origine dentaire. Pour les cellulites cutanées d'extension modérée, il faut tenir compte de la présence des staphylocoques. L'association oxacilline + métronidazole semble adéquate. La clindamycine a été très utilisée notamment en cas d'allergie aux pénicillines; on peut lui reprocher une perte d'activité sur les anaérobies puisque $20 \%$ des souches y sont résistantes, son absence d'activité sur Eikenella corrodens et le risque éventuel de colite pseudomembraneuse. Faut-il l'associer au métronidazole pour renforcer son activité sur les anaérobies résistants à la clindamycine?

C'est l'association amoxicilline + acide clavulanique qui possède le spectre le plus large.

\section{Infections sévères}

Dans les infections sévères (Tab. 7), la couverture des bacilles à Gram négatif aéro-anaérobies facultatifs n'est pas nécessaire sauf si des données bactériologiques l'indiquent. L'attente d'un résultat favorable est-elle possible face à une infection sévère? Les cliniciens préfèrent employer amoxicilline + acide clavulanique ou pipéracilline + tazobactam assurant avec cette dernière une meilleure activité sur les entéro- 
Tableau 6: Spectre des antibiotiques employés dans le traitement des cellulites modérées Activity of the antibiotics prescribed in the treatment of moderate cellulitis

\begin{tabular}{|c|c|c|c|c|}
\hline Antibiotiques & Streptocoques & $\begin{array}{l}\text { Anaérobies à } \\
\text { Gram négatif }\end{array}$ & Staphylocoques & $\mathrm{BGN}^{*}$ \\
\hline Pénicilline G & +++ & $+/ 0$ & 0 & 0 \\
\hline Amoxicilline & +++ & $+/ 0$ & 0 & 0 \\
\hline Amoxicilline + métronidazole & +++ & +++ & 0 & 0 \\
\hline Oxacilline & ++ & 0 & +++ & 0 \\
\hline Oxacilline + métronidazole & ++ & +++ & +++ & 0 \\
\hline Macrolides & ++ & + & + & 0 \\
\hline Spiramycine + métronidazole & ++ & +++ & + & 0 \\
\hline Clindamycine & ++ & ++ & ++ & 0 \\
\hline Amoxicilline + acide clavulanique & +++ & +++ & ++ & ++ \\
\hline
\end{tabular}

bactéries et éventuellement sur Pseudomonas aeruginosa. Les céphalosporines de troisième génération injectables, de type céfotaxime et ceftriaxone, ont une activité supérieure sur les entérobactéries, mais sont insuffisamment actives sur les anaérobies: elles devront donc être associées au métronidazole. En cas de résistance des entérobactéries à l'antibiogramme ou si le pronostic vital est engagé, on pourra recourir à l'imipénème.

En cas d'allergie aux B-lactamines, on peut envisager l'association de la clindamycine ou d'une fluoroquinolone à activité anaérobie comme la moxifloxacine, à un aminoglycoside. Dans les cellulites sévères, la règle est d'associer tous les antibiotiques précédemment cités à un aminoglycoside. Si ces derniers ne sont pas utilisables (insuffisance rénale sévère), la rifampicine peut être associée si la sensibilité à cet antibiotique des bactéries isolées le permet.

\section{Cas particuliers}

II reste deux situations plus rares: la présence d'un staphylocoque résistant à la méthicilline ou celle de $P$. aeruginosa.

Lorsqu'un staphylocoque est résistant à la méthicilline, il est résistant à toutes les B-lactamines. Cependant, si la souche est sensible à la fosfomycine, il est possible d'employer l'association céfotaxime + fosfomycine également active sur les entérobactéries mais inactive sur les anaérobies; on ajoutera dans ce cas le métronidazole. Une autre alternative consiste à prescrire un glycopeptide (vancomycine ou teicoplanine) que l'on associera si possible à la gentamicine. En cas d'insuffisance rénale, on peut remplacer l'aminoglycoside par la rifampicine ou une fluoroquinolone sur antibiogramme. Pour les cellulites, il faudra toujours être actif simultanément sur les anaérobies ce que permet généralement la rifampicine mais pas les fluoroquinolones sauf la moxifloxacine ou la gatifloxacine. L'emploi de la rifampicine sera prudent pour éviter la sélection de mutants résistants en monothérapie ou dans une fausse bithérapie avec résistance de la souche à l'antibiotique associé. Les effets secondaires des fluoroquinolones ne sont pas négligeables et sont à mettre en balance avec le bénéfice thérapeutique pour le malade.

Une autre solution consiste à employer le linézolide qui est actif sur les staphylocoques multirésistants, les streptocoques et les anaérobies stricts [17]. Cette oxazolidinone peut être associée à la rifampicine si l'on désire une bithérapie permettant ultérieurement un relais par voie orale. Pseudomonas aeruginosa fait l'objet d'un traitement par bithérapie. On utilise les B-lactamines anti-pyocyaniques (ticarcilline ou pipéracilline médecine

buccale

chirurgie

buccale

VOL. $11, \mathrm{~N}^{\circ} 1$ 2005

page 13 
Tableau 7: Spectre des antibiotiques employés dans le traitement des cellulites sévères Activity of the antibiotics prescribed in the treatment of severe cellulitis

\begin{tabular}{|c|c|c|c|c|}
\hline Antibiotiques & Streptocoques & $\begin{array}{l}\text { Anaérobies } \\
\text { à Gram négatif }\end{array}$ & Staphylocoques & $B G N^{*}$ \\
\hline $\begin{array}{l}\text { Amoxicilline }+ \text { acide } \\
\text { clavulanique } \pm \text { aminoglycoside }\end{array}$ & +++ & +++ & ++ & ++ \\
\hline $\begin{array}{l}\text { Pipéracilline + tazobactam } \\
\pm \text { aminoglycoside }\end{array}$ & +++ & +++ & ++ & +++ \\
\hline Ceftriaxone & +++ & + & + & +++ \\
\hline Métronidazole & 0 & +++ & 0 & 0 \\
\hline $\begin{array}{l}\text { Ceftriaxone + métronidazole } \\
+ \text { aminoglycoside }\end{array}$ & +++ & +++ & ++ & +++ \\
\hline Imipénème + aminoglycoside & +++ & +++ & ++ & +++ \\
\hline \multicolumn{5}{|c|}{ Alternatives en absence de BGN aérobies ou/et en présence de staphylocoques méti-R } \\
\hline $\begin{array}{l}\text { Céfotaxime + fosfomycine } \\
+ \text { métronidazole }\end{array}$ & +++ & +++ & +++ & +++ \\
\hline Linézolide & +++ & +++ & +++ & 0 \\
\hline Glycopeptides & +++ & 0 & +++ & 0 \\
\hline \multicolumn{5}{|c|}{ Alternative en cas d'allergie aux ß-lactamines } \\
\hline Moxifloxacine & +++ & ++ & ++ & +++ \\
\hline \multicolumn{5}{|c|}{ Antibiotiques pouvant être associés dans le cadre d'une bi -ou d'une trithérapie } \\
\hline Rifampicine $^{\star \star}$ & +++ & +++ & ++ & ++ \\
\hline Aminoglycosides & $\begin{array}{c}\text { En association avec } \\
\text { une B-lactamine } \\
\text { ou un glycopeptide }\end{array}$ & 0 & ++ & +++ \\
\hline \multicolumn{5}{|c|}{$\begin{array}{l}+++>90 \% \text { des souches sont sensibles, ++ activité inférieure, + résistance }>20 \%, \text { O pas d'activité } \\
+++ \text { more than } 90 \% \text { of strains are susceptible, ++ antibiotic less potent, + resistance }>20 \% \text {, O lack of activity } \\
\text { * BGN bacilles à Gram négatif aéro-anaérobies facultatifs } \\
\text { * BGN gram-negative aerobic bacilli } \\
\text { ** jamais en monothérapie } \\
\text { ** no use in monotherapy }\end{array}$} \\
\hline
\end{tabular}

seules ou associées à des inhibiteurs de ß-lactamase, ceftazidime, céfépime et imipénème) en association avec l'amikacine, ou à défaut la ciprofloxacine. Si une céphalosporine est employée, elle ne couvre pas les anaérobies stricts, on ajoutera donc du métronidazole.

Il peut arriver, au cours d'infections sévères, d'avoir un échec clinique dont la documentation montre que les mêmes anaérobies stricts isolés lors du premier prélèvement sont encore présents, tout en ayant gardé leur sensibilité aux molécules prescrites (amoxicilline + clavulanate, pipéracilline + tazobactam, imipénème). Ces infections graves nécessitent quelquefois une bithérapie pour les anaérobies, et en dépit de la redondance des spectres, il convient parfois de leur ajouter du métronidazole. En cas d'impasse thérapeutique sur les anaérobies, le thiamphénicol a gardé une excellente activité sur ces bactéries.

En conclusion, les cellulites sont rarement traitées en monothérapie et elles nécessitent même parfois l'adjonction d'un troisième antibiotique en respectant les règles d'association pour les antibiotiques. Cette association permet d'élargir le spectre, de renforcer la bactéricidie, d'obtenir une meilleure réponse clinique dans les infections sévères ou chez des patients neutropéniques ou immunodéprimés. Afin de respecter l'écologie microbienne, il existe une hiérarchie dans les prescriptions de ces antibiotiques qu'il convient d'apprécier selon le patient et la gravité de la cellulite. 


\section{RÉFÉRENCES}

1 - Agence Française de Sécurité Sanitaire des Produits de santé (AFSSAPS). Prescription des antibiotiques en odontologie et stomatologie. Recommandations et argumentaire. AFSSAPS, 2001.

2 - BerINI L, BRESCO M, GAY C. Buccal and cervicofacial cellulitis. Concept, pathogenesis, clinical manifestations and management. Medicina Oral 1999; 4 : 337 50 .

3 - FUJIYOSHI T, OKASAKA T, YOSHIDA M, MASHIMA K. Clinical and bacteriological significance of the Streptococcus milleri group in deep neck abscesses. Nippon Jibiinkoka Kaiho 2001; 104: 147-56.

4 - Perl B, Gottherer NP, Raveh D, Sclesinger Y, Rudensky B, YINNON AM. Cots-effectiveness of blood cultures for adult patients with cellulitis. Clin Inf Dis 1999; 29 : 14838.

5 - Eick S, Pfister W, Korn-Stemme S, MagdefesselSchumtzer U, Straube E. Pathogen and resistance spectrum in intraoral infections of the jaw-facial area with special reference to anaerobic bacteria. Mund Kiefer Gesischtschir 2000; 4 : 234-9.

6 - Brook I. Infections of the head and neck. In Pediatric anaerobic infection, diagnosis and management, $2^{\text {nd }} \mathrm{ed}$, (pp181-202). Mosby, St Louis 1989.

7 - Brook I, Frazier EH. Clinical features and aerobic and anaerobic microbiological characteristics of cellulitis. Arch Surg 1995; 130: 786-92.

8 - Kuriyama T, Karasawa T, Nakagawa K, Yamamoto E, NAKAMURA S. Incidence of B-lactamase and antimicrobial susceptibility of anaerobic gram-negative rods isolated from pus specimens of orofacial odontogenic infections. Oral Microbiol Immunol 2001; 16:10-15.

9 - Fosse T, Madinier I, Hannoun L, Giraud-Morin C, Hitzig C, CharBiT Y, OURANG S. High prevalence of cfXA ß-lactamase in aminopenicillin - resistant Prevotella strains isolated from periodontal pockets. Oral Microbiol Immunol $2002 ; 17: 85-88$.
10 - Milazzo I, Blandino G, Caccano F, Musumeci R, NicolettI G, Speciale A. Faropenem, a new oral penem: antibacterial activity against selected anaerobic and fastidious periodontal isolates. J Antimicrob Chemother 2003; 51:721-25.

11 - EICK S, Pfister W, Straube E. Antimicrobial susceptibility of anaerobic and capnophilic bacteria isolated from odontogenic abscesses and rapidly progressive periodontitis. Int J Antimicrob Agents 1999; 12:41-46.

12 - Dubreuil L, Behra-Miellet J, Vouillot C, Bland S, Sedallian A, Mory F. B-lactamase production in Prevotella and in vitro susceptibilities to selected betalactam antibiotics Int. J Antimicrob Agents 2003; 21 : 267-73.

13 - Comité de l'Antibiogramme de la Société Française de Microbiologie Report 2003. Int J Antimicrob Agent $2003 ; 21: 364-91$.

14 - NyFORS S, KÖNONEN E, SyRJÄnEN R, Komulainen E, JOUSIMIES-SOMER H. Emergence of penicillin resistance among Fusobacterium nucleatum populations of commensal oral flora during early childhood. J Antimicrob Chemother 2003; 51: 107-12.

15 - Kuriyama T, NaKagaWa K, Karasawa T, SaIKI Y, Yamamoto E, NAKAMURA S. Past administration of beta-lactam antibiotics and increase in the emergence of beta-lactamase-producing bacteria in patients with orofacial odontogenic infections. Oral Surg Oral Med Oral Pathol Radiol Endod 2000; 89: 186-92.

16 - Dubreuil L, Calvet L, Rocques C, Sednaoui P. Etat de la sensibilité aux antibiotiques des bactéries anaérobies isolées lors d'infections odontogènes. Intérêt de l'association spiramycine-métronidazole. Méd buccale Chir buccale 2003; 9: 167-76.

17 - Behra J, Calvet L, DubreuIL L. Activity of linezolid against anaerobic bacteria. Int J Antimicrob Agents 2003 ; $22: 28-34$

\begin{tabular}{l}
$\begin{array}{l}\text { médecine } \\
\text { buccale } \\
\text { chirurgie } \\
\text { buccale }\end{array}$ \\
\hline VOL. 11, N ${ }^{\circ} 1$ \\
2005 \\
\hline page 15
\end{tabular}

page 15 